\title{
QUALIDADE FÍSICO-QUÍMICA E MICROBIOLÓGICA DE HORTALIÇAS DESIDRATADAS AO SOL E EM SECADOR LABORATORIAL
}

\author{
A. S. SANTOS FILHO ${ }^{1}$, N. C. VELOSO ${ }^{1}$, R. T. CARELI ${ }^{1}$, M. N. CANO-CHAUCA ${ }^{1}$, C. A. COSTA $^{1}$, \\ N. J. F. OLIVEIRA ${ }^{1}$, J. A. CAMPOS $^{2}$ \\ ${ }^{1}$ Universidade Federal de Minas Gerais - UFMG, ${ }^{2}$ Universidade Federal de Viçosa - UFV \\ adairof@gmail.com ${ }^{*}$
}

Submetido 10/02/2018 - Aceito 21/10/2018

DOI: $10.15628 /$ holos.2018.6922

\begin{abstract}
RESUMO
Em Minas Gerais, Brasil, couve e Ora-pro-nóbis são folhosas comuns e a dessecação dessas pode aumentar vida útil e valor agregado. Objetivou-se desidratar e realizar análise físico-química e microbiológica de Brassica oleraceae e Pereskia aculeata ao sol e em secador. Folhas sanitizadas e enxaguadas foram cortadas em espessura aproximada de $5 \mathrm{~mm}$ e porções de $300 \mathrm{~g}$ foram colocadas em bandejas, no secador a 60 ${ }^{\circ} \mathrm{C}$ e ao sol. Monitorou-se a perda de umidade até estabilização da massa e elaboraram-se gráficos de secagem. Após estabilizar por 8h, as amostras foram acondicionadas a vácuo e armazenadas em temperatura ambiente por cinco dias para análises físicas, microbiológicas e químicas. A perda de massa foi mais acentuada nas primeiras horas e posteriormente observou-se decréscimo. As equações de perda de massa foram: $y=5,649 x^{2}-77,63 x+301,0$ para couve
\end{abstract}

cortada sob secagem ao sol; $y=2,800 x^{2}-53,35 x+300,2$ para Ora-pro-nóbis cortado sob secagem ao sol nas primeiras $10 \mathrm{~h} ; \mathrm{y}=0,098 \mathrm{x}^{2}-2,099 \mathrm{x}+40,30$ para Ora-pronóbis cortado sob secagem ao sol nas $4 \mathrm{~h}$ seguintes; $y=1,449 x^{2}-39,50 x+300$ para couve cortada em secagem artificial; $y=1,637 x^{2}-42,93 x+300$ para Ora-pro-nóbis cortado sob secagem artificial; $y=0,377 x^{2}-20,02 x+293,0$ para folhas íntegras de Ora-pro-nóbis sob secagem artificial. Constatou-se ausência de contaminantes físicos e de Salmonella spp., além de contagens de Staphylococcus coagulase positivo e coliformes a $35^{\circ} \mathrm{C}$ inferiores às permitidas, nos dois métodos de desidratação. As médias de umidade foram 8,32 a 8,37\% e de proteínas, 18,72 a $24,74 \%$. A qualidade físicoquímica e microbiológica de $B$. oleraceae e $P$. aculeata desidratadas ao sol e em secador foi satisfatória.

PALAVRAS-CHAVE: Processamento mínimo, Folhosa, Olerícola, Hortaliça não convencional, Lobrobô.

\section{PHYSICAL AND CHEMICAL QUALITY AND MICROBIOLOGICAL ANALYSIS OF VEGETABLES DEHYDRATED IN THE SUN AND IN LABORATORY DRYER}

\begin{abstract}
In Minas Gerais, Brazil, cabbage and ora-pro-nóbis are common hardwoods and it is conserved by desiccation can increase the shelf life and value. This study aimed to dehydrate Brassica oleraceae and Pereskia aculeata by sunlight and in laboratory dryer and determine its physicalchemical and microbiological quality. The leaves disinfected were cut to a thickness of $5 \mathrm{~mm}$ and portions of $300 \mathrm{~g}$ were placed on dryer at $60^{\circ} \mathrm{C}$ and under the sun. The loss of moisture has monitored until stabilization of the weight and graphics were designed with mass variation. After humidity stabilization for $8 \mathrm{~h}$, the samples were vacuum packaging and stored at room temperature for five days to physical, microbiological and chemical tests. The mass loss was more accentuated in first hours and subsequently decreased. The mass loss equations were: $y=5.649 x^{2}-$
\end{abstract}

$77.63 x+301.0$ for cut cabbage under sun drying; $y=2.800 x^{2}-$ $53.35 x+300.2$ for cut Ora-pro-nóbis under sun drying in the first $10 h ; y=0.098 x^{2}-2,099 x+40.30$ for cut Ora-pro-nóbis under sun drying in following $4 h ; y=1.449 x^{2}-39,50 x+300$ for cut cabbage into artificial drying; $y=1.637 x^{2}-42.93 x+300$ for cut Ora-pro-nóbis under artificial drying; $y=0,377 x^{2}-$ $20,02 x+293,0$ for uncut leaves of Ora-pro-nóbis under artificial drying. It was not physical contaminants and Salmonella spp., besides counts of Coagulase positive Staphylococcus. and coliforms at $35^{\circ} \mathrm{C}$ below the allowed, for the two methods of dehydration. The humidity mean in dehydrated vegetables were 8.32 to $8.37 \%$ and proteins, 18.72 to $24.74 \%$. The physical-chemical and microbiological quality of dehydrated $B$. oleraceae and $P$. aculeata under sunlight and in artificial dryer were satisfactory.

KEYWORDS: Minimal processing, Hardwood, Vegetable crop, Unconventional vegetable, Barbados gooseberry. 


\section{INTRODUÇÃO}

A couve, Brassica oleracea, é originária do Mediterrâneo, cultivada todo o ano e em diversas regiões do Brasil. Nutricionalmente é rica em minerais, em especial cálcio e ferro, além de fibras e vitaminas (Azevedo et al., 2016; Brandão et al., 2015; Pigoli, 2012). Ora-pro-nóbis, Pereskia aculeata, é cactácea nativa da América Latina e dos Estados Unidos, descrita na África e Inglaterra. O teor proteico superior a $20 \%$ e a presença de aminoácidos essenciais utilizando-se para enriquecimento de dietas (Almeida \& Corrêa, 2012; Almeida Filho \& Cambraia, 1974; Paterson et al., 2009; Takeiti et al., 2009) e o uso como analgésico, dentre outros usos fitoterápicos tradicionais (Zareisedehizadeh, Tan \& Koh, 2014; Pinto et al., 2015). Em Minas Gerais o consumo é realizado na culinária há décadas (Pompeu et al., 2014).

A perda pós-colheita de hortaliças é elevada, sendo maior com manipulação e conservação incorretas. Para reduzir esse prejuízo e manter os atributos mais próximos possíveis ao alimento in natura são importantes técnicas de conservação. Estas, em folhosas, tendem a assegurar a qualidade do produto final, podem gerar menor descarte (Barbosa et al., 2015) e são utilizadas para diversos alimentos (Rocha et al., 2008).

A desidratação é um dos métodos mais antigos para conservar alimentos e quando utilizada de maneira correta, permite manter a maioria das propriedades nutricionais. $\mathrm{A}$ umidade eliminada por evaporação restringe a água livre e pode reduzir a presença de micro-organismos deteriorantes. A secagem de vegetais pode aumentar a vida útil, agregar valor ao produto final e ser realizada de forma natural ou artificial, se feita ao sol ou em secador, respectivamente (Sanjinez-Argandoña et al., 2011). O teor de umidade de vegetais desidratados variou entre 7,53 a 17,65\% (Silva et al., 2008). Porém, se mal processados e/ou acondicionados inadequadamente, alimentos desidratados podem apresentar pior qualidade (Rodríguez \& Pérez, 2015).

A adição de hortaliça desidratada pode facilitar o processamento e evitar a rejeição dessa, especialmente por crianças, sendo possível acrescentar o vegetal em misturas mais palatáveis para permitir o balanceamento de dieta infantil (Rocha et al., 2008). Mas antes de inserir esse produto no mercado é preciso realizar análises microbiológicas estabelecidas na legislação, as quais garantem a qualidade final e evitam a transmissão de doenças (Oliveira et al., 2013).

Massa de macarrão acrescida de $P$. aculeata desidratada foi bem aceita por consumidores e os nutrientes foram preservados, evidenciando potencial uso como fonte alimentar saudável (Rocha et al., 2008). Apesar de couve e Ora-pro-nóbis serem de consumo tradicional em Minas Gerais (Almeida \& Corrêa, 2012; Brandão et al., 2015), estudos desses vegetais desidratados são escassos na literatura, possivelmente por causa do rendimento pequeno de ambos após a secagem e por dificuldades de cultivo e de manuseio, especialmente de $P$. aculeata, considerada por Takeiti et al. (2009) como hortaliça não convencional. Portanto, objetivou-se desidratar $B$. oleraceae e $P$. aculeata ao sol e em secador de circulação forçada de ar, determinar a qualidade físico-química e microbiológica do produto final. 


\section{MATERIAL E MÉTODOS}

Os vegetais foram processados no Laboratório de Ciência e Tecnologia de Alimentos do Instituto de Ciências Agrárias da Universidade Federal de Minas Gerais (ICA/UFMG). Selecionaramse folhas íntegras e posteriormente essas foram lavadas em água corrente, sanitizadas em solução 0,5\% (m/v) de desinfetante dicloroisocianato de sódio para hortifrutícolas por $10 \mathrm{~min}$ e enxaguadas em água potável para retirar os resíduos de bactericida. $O$ excesso de água foi removido com centrífuga manual por um minuto.

O processamento mínimo consistiu-se de cortes manuais em espessura aproximada de 5 $\mathrm{mm}$, antes de iniciar a desidratação. Foram utilizadas facas de aço inoxidável afiadas, por constituírem material de fácil higienização, minimizando a contaminação microbiana durante este procedimento, além de o corte preciso reduzir os danos mecânicos as hortaliças (Vitti et al., 2004). A matéria prima foi dividida em parcelas de $300 \mathrm{~g}$ em cada bandeja de $30 \times 40 \times 1 \mathrm{~cm}$ do secador e colocada ao sol sobre mesa de aço inoxidável sem cobertura, a fim de facilitar a secagem.

A desidratação artificial foi conduzida em secador com circulação forçada de ar, e termostato acoplado ao sistema de aquecimento para estabilização automática da temperatura interna em $60^{\circ} \mathrm{C}$. Somente após atingir esse valor inseria-se o vegetal nas bandejas em quantidade igual à experiência ao sol.

A taxa de perda da umidade foi monitorada com pesagem em balança digital a cada duas horas para a experimentação ao sol e a cada oito horas no experimento com secador, até haver pelo menos duas pesagens sucessivas constantes. Foram elaborados gráficos (Flemming \& Gonçalves, 2006) de secagem em função da variação da massa com o programa Excel (EXCEL, 2007).

Ao término do processamento, para estabilizar a umidade do produto, as amostras permaneceram em bandejas de aço inox por oito horas em bancadas do laboratório e foram protegidas com tela de nylon. Em seguida, foram acondicionadas em embalagens plásticas, fechadas a vácuo, identificadas e armazenadas em temperatura ambiente por cinco dias, quando se iniciaram os testes físico-químicos, de acordo metodologia do Instituto Adolfo Lutz (Zenebon et al., 2008) e microbiológicos, segundo descrito por Silva et al. (2010).

\section{RESULTADOS E DISCUSSÃO}

Em todos os processos a perda de massa mais acentuada ocorreu nas primeiras horas (Figura 1, Figura 2, Figura 4, Figura 5 e Figura 6). Nesse período, a transferência de massa e de calor foi equivalente. Consequentemente, a velocidade de secagem tenderá a constância, em decorrência da quantidade suficiente de água na superfície do produto para acompanhar a evaporação, conforme descreveram Park et al. (2001). Após as primeiras horas foi possível observar o decréscimo de secagem (Figura 2, Figura 3, Figura 4, Figura 5 e Figura 6), pois, conforme Park et al. (2001) a energia na forma de calor fornecida ao alimento foi insuficiente para retirar a mesma quantidade de água perdida inicialmente. O término da secagem ocorreu quando o produto atingiu a umidade de equilíbrio. 


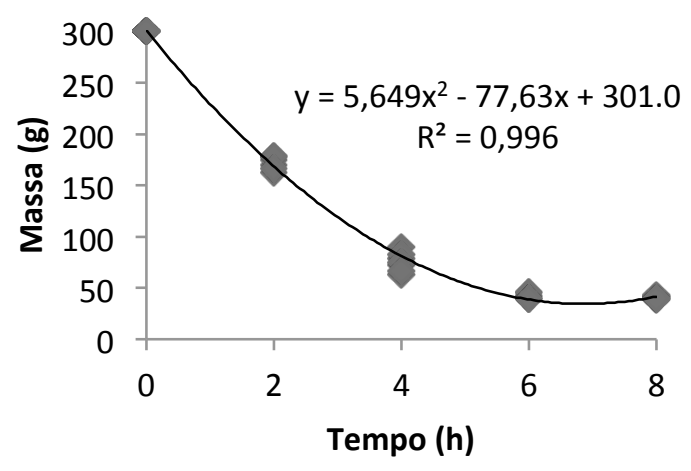

Figura 1: Perda de massa de folhas cortadas de couve-manteiga no processo de secagem natural ao sol em função do tempo de secagem.

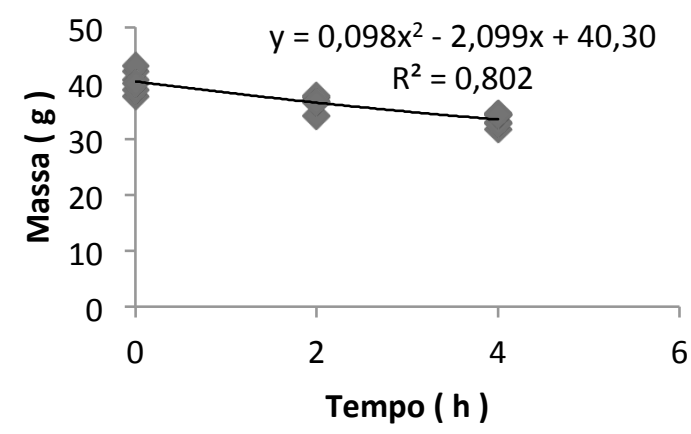

Figura 3: Perda de massa de folhas cortadas de Ora-pro-nóbis no processo de secagem natural ao sol nas $\mathbf{4}$ h seguintes (dia 2 ).

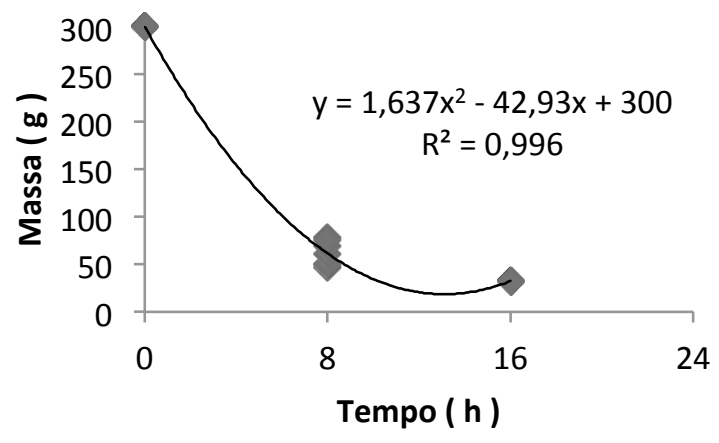

Figura 5: Perda de massa de folhas cortadas de Ora-pro-nóbis no processo de secagem artificial em função do tempo de secagem.

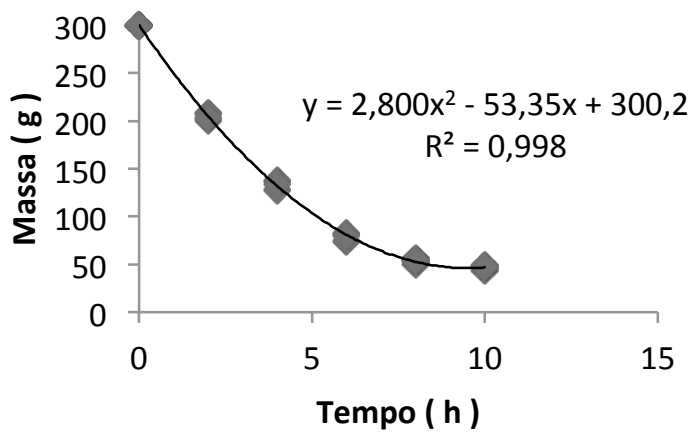

Figura 2: Perda de massa de folhas cortadas de Ora-pro-nóbis no processo de secagem natural ao sol nas primeiras $10 \mathrm{~h}$ (dia 1).

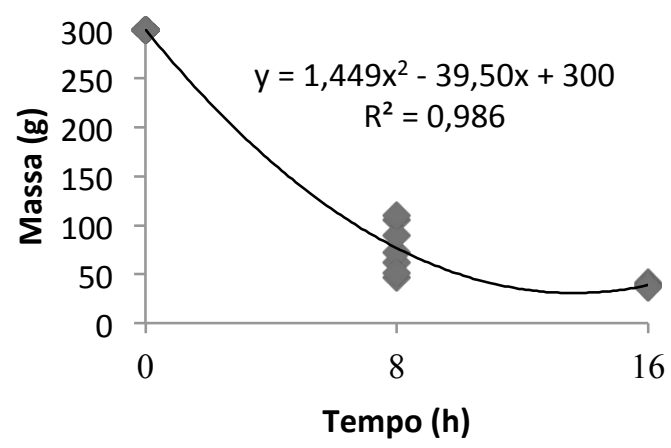

Figura 4: Perda de massa de folhas cortadas de Couve Manteiga no processo de secagem artificial em função do tempo de secagem.

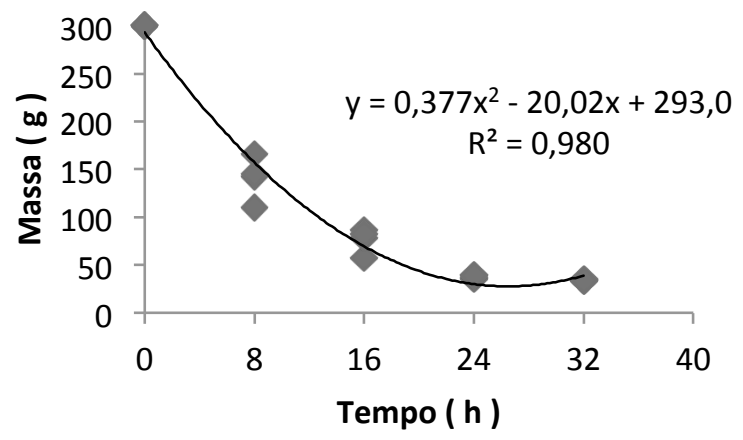

Figura 6: Perda de massa de folhas íntegras de Ora-pro-nóbis no processo de secagem artificial em função do tempo de secagem. 
As equações de perda de massa das Figuras 1, 2 e 3 foram: $y=5,649 \times 2-77,63 x+301,08$ e $R^{2}=0,996$ para couve cortada sob secagem ao sol; $y=2,800 \times 2-53,35 x+300,2$ e $R^{2}=0,998$ para de Orapro-nóbis cortado sob secagem ao sol nas primeiras $10 \mathrm{~h}$ (dia 1) e $\mathrm{y}=0,098 \times 2-2,099 x+40,30$ e $\mathrm{R}^{2}=0,802$ para Ora-pro-nóbis cortado sob secagem ao sol nas $4 \mathrm{~h}$ seguintes (dia 2). $\mathrm{A}$ experimentação com couve-manteiga aconteceu somente em um dia (Figura 1), pois a energia fornecida foi suficiente para retirar a umidade do produto nesse tempo. A velocidade de transferência de massa foi confirmada ao comparar as equações da velocidade de secagem dos alimentos, na qual a função da hortaliça convencional possui maior coeficiente angular. A desidratação natural ao sol de $P$. aculeata precisou ser realizada em dois dias (Figura 2 e Figura 3 ), porque a energia solar foi insuficiente para retirar toda a umidade do alimento até a estabilização completa da massa, sendo necessárias mais quatro horas de exposição solar no dia seguinte. A maior velocidade no primeiro dia é evidenciada na verificação da equação da reta em função do tempo a qual obteve maior coeficiente angular.

A secagem natural de folhas íntegras de Ora-pro-nóbis foi inviabilizada porque a ocorrência do vento foi suficiente para que essas fossem lançadas ao solo, sendo contaminadas. Possivelmente, a altura de $1 \mathrm{~cm}$ das bandejas favoreceu essa situação, apesar das bordas da mesa de aço inox possuírem $7 \mathrm{~cm}$ de altura. A desidratação natural pode possuir menor custo em relação à desidratação artificial, apesar de demandar mais tempo e depender das condições climáticas, segundo Silva et al. (2008) e do maior risco de contaminação, conforme confirmado nesse experimento com as folhas íntegras.

As equações de perda de massa das Figuras 4, 5 e 6 foram: $y=1,6378 x^{2}-42,933 x+300$; $y=0,3777 x^{2}-20,023 x+293,04$ e $y=1,449 x^{2}-39,505 x+300$. De acordo com as funções de velocidade referentes à secagem das hortaliças cortadas (Figuras 4 e 5 ) o processamento de Ora-pró-nobis foi mais acelerado, pois o coeficiente angular ultrapassa o de $B$. oleracea. Este, porém possui maior eficiência de secagem, se comparado a $P$. aculeta íntegra (Figura 6).

Nas Figuras 7 e 8 são demonstrados o aspecto fotográfico das folhas íntegras e fatiadas de Ora-pró-nobis após desidratação artificial em secador laboratorial. As folhas alteraram a cor natural de verde brilhante para a coloração verde musgo. Variações de cor ocorrem em qualquer vegetal ao ser desidratado, conforme Silva et al. (2008) e Park et al. (2001).

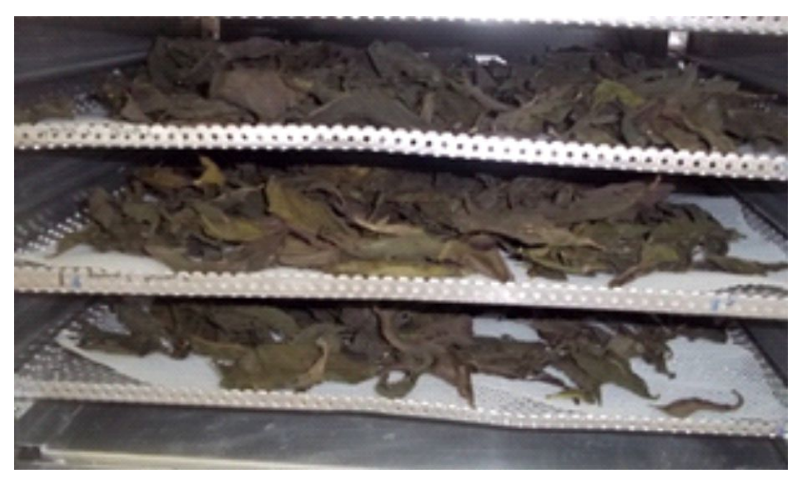

Figura 7: Fotografia do aspecto de folhas íntegras de Ora-pro-nóbis após secagem artificial.

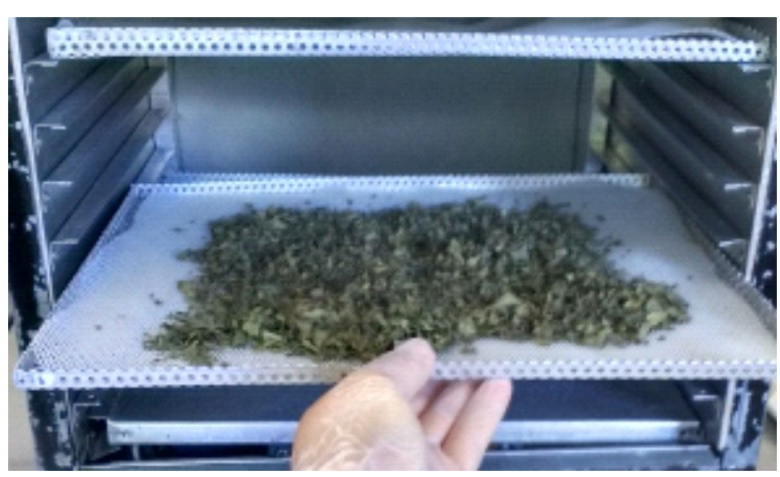

Figura 8: Fotografia do aspecto de folhas cortadas de Ora-pro-nóbis após secagem artificial. 
Observaram-se, aproximadamente 16 horas como suficientes para desidratar artificialmente as hortaliças fatiadas (Figura 8). Ao visualizar as Figuras 5 e 6 observou-se maior perda de massa das folhas cortadas em relação às íntegras, pois as equações de velocidade apresentaram coeficientes angulares maiores, diretamente proporcionais à variação da massa em função do tempo. Essa diferença aconteceu porque as folhas cortadas oferecem maior superfície de contato para o ar e possuem células rompidas por causa do processamento mínimo. Essas características beneficiam a melhor transferência de calor no período de tratamento, conforme observado por Sanjinez-Argandoña et al. (2011).

As análises físicas demonstraram ausência de contaminantes no alimento. Portanto, os procedimentos higiênicos adotados no período de manuseio do alimento foram eficientes. Como pode ser visualizado na Tabela 1, no teste microbiológico verificou-se ausência de Salmonella spp. As contagens de Staphylococcus coagulase positivo e coliformes foram inferiores às máximas permitidas. Assim, essas amostras poderiam ser utilizadas para enriquecer misturas de massas e outros alimentos, da mesma forma que realizado por Rocha et al. (2008), quando desenvolveram massa de macarrão adicionada de Ora-pro-nóbis desidratado. Entretanto, para assegurar a qualidade sanitária do produto final, conforme descrito por Oliveira et al. (2013) é preciso realizar análises microbiológicas estabelecidas na legislação.

Tabela 1. Resultados microbiológicos de Couve manteiga e Ora-pro-nóbis desidratado por diferentes métodos.

\begin{tabular}{ccccccc}
\hline Micro-organismo & $\begin{array}{c}\text { *Tolerância } \\
\text { por amostra } \\
\text { indicativa }\end{array}$ & $\begin{array}{c}\text { B. oleracea } \\
\text { desidratada } \\
\text { naturalmente }\end{array}$ & $\begin{array}{c}\text { P.aculeata } \\
\text { desidratada } \\
\text { naturalmente }\end{array}$ & $\begin{array}{c}\text { B. } \\
\text { oleracea } \\
\text { cortada }\end{array}$ & $\begin{array}{c}P . \\
\text { aculeata } \\
\text { íntegra }\end{array}$ & $\begin{array}{c}P . \\
\text { aculeata } \\
\text { cortada }\end{array}$ \\
\hline $\begin{array}{c}\text { Salmonella spp. } \\
\text { (prevalência em } \\
25 \text { g) }\end{array}$ & Ausente & Ausente & Ausente & Ausente & Ausente & Ausente \\
$\begin{array}{c}\text { Staphylococcus } \\
\text { coagulase positivo } \\
\text { (UFC.g }{ }^{-1} \text { ) }\end{array}$ & $1,0 \times 10^{2}$ & $<1,0 \times 10^{1}$ & $<1,0 \times 10^{1}$ & $<1,0$ & $<1,0$ & $<1,0$ \\
$\begin{array}{c}\text { Coliformes a } 45^{\circ} \mathrm{C} \\
\text { (NMP.g }{ }^{-1} \text { ) }\end{array}$ & $1,0 \times 10^{3}$ & $1,5 \times 10^{1}$ & $<3,0$ & 9,2 & 9,2 & $1,5 \times 10^{1}$ \\
\hline
\end{tabular}

UFC. $\mathrm{g}^{-1}=$ Unidades Formadoras de Colônia por g; NMP.g ${ }^{-1}=$ Número mais provável por grama. *Valores de referência conforme Resolução de Diretoria Colegiada n 12, de 2 de janeiro de 2001 (BRASIL, 2001).

Resultado semelhante, ausência de Salmonella spp. foi descrito em cenoura, beterraba e espinafre desidratados artificialmente por Zanatta et al. (2010). Staphylococcus coagulase positivo (UFC. ${ }^{-1}$ ) identificados na presente pesquisa foram compatíveis com os valores permitidos (BRASIL, 2001). A estimativa de Coliformes a $45^{\circ} \mathrm{C}$ em $P$. aculeata desidratada naturalmente, inferior a 3 NMP.g $g^{-1}$ é igual ao verificado por Zanatta et al. (2010) em cenoura, beterraba e espinafre desidratados. 
Seguir técnicas de processamento adequadas durante a manipulação e preparação de alimentos é imprescindível, pois, segundo os autores anteriormente citados, a quantidade desses contaminantes pode definir a qualidade final e os prováveis danos à saúde do consumidor. Não foram observadas diferenças de contaminação por micro-organismos entre os métodos natural ou artificial, considerando-se o limite máximo da legislação. Portanto, se realizados de forma adequada, ambos podem garantir folhosas desidratadas aptas para o consumo humano.

Os processos de secagem utilizados na experimentação podem ser considerados eficientes, pois os teores médios de umidade dos vegetais desidratados, de $8,32 \%$ para $B$. oleracea e $8,37 \%$ para $P$. aculeata (Tabela 2) apresentam-se dentro dos limites de 7,53 a $17,65 \%$ de umidade, encontrados por Silva et al. (2008). A perda de água intensifica a concentração de nutrientes e é importante para valorizar a matéria prima, ao permitir utilizá-la como alimento semi preparado com composição nutricional melhorada, como descrito por Sanjinez-Argandoña et al. (2011).

Tabela 2. Composição centesimal e valores médios do experimento em base de matéria seca de Couve-manteiga e Ora-pro-nóbis desidratados em comparação com a literatura disponível

\begin{tabular}{|c|c|c|c|c|c|c|}
\hline \multirow[b]{2}{*}{ Vegetal } & \multirow[b]{2}{*}{$\begin{array}{c}\text { Método } \\
\text { de } \\
\text { Secagem }\end{array}$} & \multirow[b]{2}{*}{$\begin{array}{l}\text { Umidad } \\
\text { e }\end{array}$} & \multicolumn{4}{|c|}{ Composição nutricional (\%) } \\
\hline & & & Minerais & $\begin{array}{c}\text { Proteína } \\
\text { s }\end{array}$ & Lipídios & $\begin{array}{c}\text { Carboidratos } \\
\text { totais }\end{array}$ \\
\hline $\begin{array}{l}\text { B. oleracea } \\
\text { desidratada }\end{array}$ & Natural & 7,81 & 12,37 & 23,64 & 4,20 & 59,79 \\
\hline $\begin{array}{l}\text { B. oleracea } \\
\text { desidratada }\end{array}$ & Artificial & 8,82 & 10,84 & 24,84 & 3,33 & 60,99 \\
\hline Média & - & 8,32 & 11,61 & 24,74 & 3,77 & 60,39 \\
\hline $\begin{array}{l}{ }^{* *} B . \text { oleracea In } \\
\text { natura }\end{array}$ & - & 90,9 & 3,2 & 2,9 & 0,6 & 7,4 \\
\hline $\begin{array}{l}\text { P. aculeata } \\
\text { desidratada/cortes }\end{array}$ & Natural & 10,09 & 14,19 & 19,90 & 4,45 & 61,46 \\
\hline $\begin{array}{l}\text { P. aculeata } \\
\text { desidratada/cortes }\end{array}$ & Artificial & 7,92 & 11,69 & 16,80 & 3,15 & 68,36 \\
\hline $\begin{array}{l}\text { P. aculeata } \\
\text { desidratada/íntegra }\end{array}$ & Artificial & 7,10 & 14,98 & 17,97 & 3,03 & 64,02 \\
\hline Média & - & 8,37 & 13,62 & 18,22 & 3,54 & 64,61 \\
\hline $\begin{array}{l}{ }^{1} P \text {. aculeata } \\
\text { Guiricema- } \mathrm{MG}^{\mathrm{a}}\end{array}$ & - & $*$ & 21,70 & 17,40 & 11,70 & 49,2 \\
\hline $\begin{array}{l}{ }^{1} P . \text { aculeata/Viçosa- } \\
\mathrm{MG}^{\mathrm{a}} \\
{ }^{1} P .\end{array}$ & - & $*$ & 21,10 & 25,50 & 6,80 & 46,6 \\
\hline $\begin{array}{l}\text { aculeata/Mairiporã- } \\
\mathrm{SP}^{\mathrm{b}}\end{array}$ & - & 89,50 & 16,10 & 28,40 & 4,10 & 51,40 \\
\hline
\end{tabular}


Observa-se na Tabela 2 alto teor proteico médio do ora-pro-nóbis (18,22\%), valor pouco inferior aos 21,85\% reportados por Souza et al. (2016) ao analisar Pereskia aculeata cultivada com doses crescentes de nitrogênio. Possivelmente por ser proveniente de extração em quintal o Orapro-nóbis coletado na presente pesquisa não foi submetido aos tratos culturais suficientes para aumentar o teor de proteína nas folhas. Em contrapartida, a couve apresentou valor de proteína médio de $24,74 \%$, provavelmente por esta ser cultivada com práticas agronômicas adequadas. A fertilização nitrogenada melhora o perfil nutricional de $P$. aculeata, conforme Souza et al. (2016). A composição nutricional de vegetais varia em função da diversidade genética das plantas, de acordo com Paterson et al. (2009) e das práticas culturais empregadas para o cultivo segundo Mazia \& Sartor (2012) e Souza et al. (2016b). Segundo Zem et al. (2017) Ora-pro-nóbis é boa fonte de proteína de qualidade. Portanto, a composição nutricional das hortaliças desidratadas reflete as características individuais e de cultivo de cada vegetal.

\section{CONCLUSÃO}

Os processos de desidratação de $B$. oleraceae e $P$. aculeata ao sol e em secador de circulação forçada de ar foram factíveis e a qualidade físico-química e microbiológica do produto final foi considerada satisfatória. Sendo aceitável utilizá-las como fonte de nutrientes para enriquecer dietas carentes em fibras e proteínas.

\section{AGRADECIMENTOS}

A Fundação de Amparo à Pesquisa do Estado de Minas Gerais (FAPEMIG) por concessão de bolsa de Iniciação Científica ao projeto e a Pro - Reitoria de Pesquisa da UFMG (PRPq - UFMG).

\section{REFERÊNCIAS}

Almeida Filho, J., \& Cambraia, J. (1974). Estudo do valor nutritivo do "Ora-pro-nóbis" (Pereskia aculeata Mill). Revista Ceres, Viçosa, 21(114), 105-111.

Almeida, M. E. F., \& Corrêa, A. D. (2012). Utilização de cactáceas do gênero Pereskia na alimentação humana em um município de Minas Gerais. Ciência Rural, Santa Maria, 42(4), 751-756.

Azevedo, A. M., Andrade Júnior, V. C., Pedrosa, C. E., Valadares, N. R., Andrade, R. F., \& Souza, J. R. S. (2016). Estudo da repetibilidade genética em clones de couve. Horticultura Brasileira, Vitória da Conquista, 34(1), 119-124.

Barbosa, C. K. R., Finger, F. L., \& Casali, V. W. D. (2015). Handling and postharvest shelf life of orapro-nobis leaves. Acta Scientiarum Agronomy, Maringá, 37(3), 307-311.

Brandão, A. A., Costa, C. A., Galizoni, F. M., Cavalcante, T. F. M., \& Neves, A. C. (2015). Perfil socioeconômico dos consumidores de hortaliças em feiras livres na microrregião de Januária. Horticultura Brasileira, Vitoria da Conquista, 33(1), 119-124.

BRASIL. (2001). Ministério da Saúde. Agencia Nacional de Vigilância Sanitária - ANVISA. Resolução RDC n. 12 de 02 jan. de 2001. Aprova o regulamento técnico sobre padrões microbiológicos para alimentos. Diário Oficial da União, Brasília, DF, 10 jan. Seção 1. 45 p. 
EXCEL. (2007). Excel : software. Versão 2007. Redmond: Microsoft, 2007.

Flemming, D. M., \& Gonçalves, M. B. (2006). Cálculo A: funções, limite, derivação e integração. 6. ed. São Paulo: Pearson Prentice Hall, 115-176.

Mazia, R. S., \& Sartor, C. F. F. P. (2012). Influência do tipo de solo usado para o cultivo de Pereskia aculeata sobre propriedade proteica. Revista Saúde e Pesquisa, Maringá, 5(1), 59-65.

NEPA - Núcleo de Estudos e Pesquisas em Alimentação. (2011). Universidade Estadual De Campinas - Unicamp. Tabela Brasileira de Composição de Alimentos (TACO), 4 ed., 161 p.

Oliveira, A. B. A., Capalonga, R., Silveira, J. T., Tondo, E. C., \& Cardoso, M. R. I. (2013). Avaliação da presença de microrganismos indicadores higiênico-sanitários em alimentos servidos em escolas públicas de Porto Alegre, Brasil. Ciência e Saúde Coletiva, Rio de Janeiro, 18(4), 955962.

Park, K. J., Yado, M. K. M., \& Brod, F. P. M. (2001). Estudo de secagem de pêra bartlett (Pyrus sp.) em fatias. Ciência e Tecnologia de Alimentos, Campinas, 21(3), 288-292.

Paterson, I. D., Downie, D. A., \& Hill, M. P. (2009). Using molecular methods to determine the origin of weed populations of Pereskia aculeata in South Africa and its relevance to biological control. Biological Control, Davis, 48(1), 84-91.

Paulino, G. C., Lucena, D. W. A., Madruga, L. C. F., Menezes, P. D. L., \& Sousa, P. P. R. (2012). Gestión de calidad del servicio de alimentos y bebidas: la importancia del manipulador de alimentos em la calidad del servicio hotelero de la ciudad de João Pessoa, Brasil. Estudios y perspectivas en turismo, Buenos Aires, 21(3), 763-777.

Pigoli, D. R. (2012). Alterações nutricionais em hortaliças decorrentes de diferentes métodos de cozimento. Dissertação de mestrado, Faculdade de Ciências Agronômicas da UNESP Campus de Botucatu, Botucatu, SP, Brasil.

Pinto, N. D. E. C., Duque, A. P., Pacheco, N. R., Mendes, R. D. E. F., Motta, E. V., Bellozi, P. M., Ribeiro, A., Salvador, M. J., \& Scio, E. (2015). Pereskia aculeata: a plant food with antinociceptive activity. Pharmaceutical Biology, Nova York, 53(12), 1-6.

Pompeu, D. G., Carvalho, A. S., Costa, O. F., Galdino, A. S., Gonçalves, D. B., Silva, J. A., \& Granjeiro, P. A. (2014). Anti-nutritional factors and "in vitro" digestibility of leaves of Pereskia aculeata Miller. Biochemistry and Biotechnology Reports, 3(1), 1-9.

Rocha, D. R. C., Pereira Júnior, G. A., Vieira, G., Pantoja, L., Santos, A. S., \& Pinto, N. A. V. D. (2008). Macarrão adicionado de Ora-pro-nóbis (Pereskia aculeata Miller) desidratado. Alimentos e Nutrição, Araraquara, 19(4), 459-465.

Rodríguez, P., \& Pérez, E. (2015). Efecto del tratamiento térmico sobre el contenido de aminoácidos de harina de plátano de dos clones. Revista lon, Bucaramanga, 28(1), 55-62.

Sanjinez-Argandoña, E. J., Branco, I. G., Bittencourt, T. U., \& Munhoz, C. L. (2011). Influência da geometria e da temperatura na cinética de secagem de tomate (Lycopersicum esculentum). Ciência e Tecnologia de Alimentos, Campinas, 31(2), 308-312.

Silva, A. S., Almeida, F. A. C., Silva, F. L. H., Dantas, H. J., \& Lima, E. E. (2008). Desidratação e efeito de pré-tratamentos no extrato seco do pimentão verde. Revista Brasileira de Produtos Agroindustriais, Campina Grande, 10(1), 27-34. 
Silva, N., Junqueira, V. C. A., Silveira, N. F. A., Taniwaki, M. H., Gomes, R. A. R., Okazaki, M. M. (2010). Manual de métodos de análise microbiológica de alimentos e água. 4. ed. São Paulo: Livraria Varela, 632 p.

Souza, M. R., Pereira, P. R. G., Magalhães, I. P. B., Sediyama, M. A. N., Vidigal, S. M., Milagres, C. S. F., \& Baracat-Pereira, M. C. (2016). Mineral, protein and nitrate contents in leaves of Pereskia aculeata subjected to nitrogen fertilization. Pesquisa Agropecuária Tropical, Goiânia, 46(1), 43-50.

Takeiti, C. Y., Antonio, G. C., Motta, E. M. P., Collares-Queiroz, F. P., \& Park, K. J. (2009). Nutritive evaluation of a non-conventional leafy vegetable (Pereskia aculeata Miller). International Journal of Food Sciences and Nutrition, Reino Unido, 60(1), 148-160.

Veit, J. C., Freitas, M. B., Reis, E. S., Moore, O. Q., Finkler, J. K., Boscolo, W. R., \& Feiden, A. (2012). Desenvolvimento e caracterização de bolos de chocolate e de cenoura com filé de tilápia do nilo (Oreochromis niloticus). Alimentos e Nutrição, Araraquara, 23(3), 427-433.

Vitti, M. C. D., Kluge, R. A., Gallo, C. R., Schiavinato, M. A., Moretti, C. L., Jacomino, A. P. 2004. Aspectos fisiológicos e microbiológicos de beterrabas minimamente processadas. Pesquisa Agropecuária Brasileira, Brasília, 39(10), 1027-1032.

Zanatta, C. L., Schlabitz, C., \& Ethur, E. M. (2010). Avaliação físico-química e microbiológica de farinhas obtidas a partir de vegetais não conformes à comercialização. Alimentos $e$ Nutrição, Araraquara, 21(3), 459-468.

Zareisedehizadeh, S., Tan, C., \& Koh, H. (2014). A Review of Botanical Characteristics, Traditional Usage, Chemical Components, Pharmacological ctivities, and Safety of Pereskia bleo (Kunth) DC. Evidence-Based Complementary and Alternative Medicine, 2014(1), 1-11.

Zem, L. M., Helm, C. V., Henriques, G. S., Cabrini, D. A., \& Zuffellato-Ribas, K. C. (2017). Pereskia aculeata: biological analysis on wistar rats. Food Science and Technology, 37(1), 42-47.

Zenebon, O., Pascuet, N. S., Tiglea, P. (2008). Métodos físico-químicos para análise de alimentos. 4. ed. São Paulo: Instituto Adolfo Lutz, 1020 p. 